\title{
Effect of Acupuncture on Carnitine for Skeletal Muscle Fatigue
}

\author{
Shizuo Toda \\ Department of Health Sciences, Kansai University of Health Sciences, Osaka, Japan \\ Email: toda@kansai.ac.jp
}

Received November 25, 2011; revised December 27, 2011; accepted January 10, 2012

\begin{abstract}
Skeletal muscle fatigue is a common symptom in various diseases, works and exercises. These were generally induced by neuron, metabolic conditions, overused muscle, and stress. But, there have been few principles about it. Many researchers have reported that acupuncture therapy has been useful to skeletal muscle fatigue on various diseases and conditions. However, it has never been shown why acupuncture therapy has the effect on skeletal muscle fatigue. The deficiency of carnitine induces fatigue, weakness, and disorder of skeletal muscle. It has showed that acupuncture induces the increase of carnitine in skeletal muscle. These findings demonstrated that acupuncture on skeletal muscle fatigue could increase carnitine as a possible affection mechanism.
\end{abstract}

Keywords: Acupuncture; Carnitine; Skeletal Muscle Fatigue

\section{Introduction}

Skeletal muscle fatigue is a common symptom experienced in various diseases, works and exercises. These were generally induced by neuron, metabolic conditions, overused muscle, and stress. But, there have been few principles about it. The study of muscle fatigue has been stated about the syndrome. Recently, many studies have reported a lot of factors. Those are calcium, potassium, phosphate, lactate, central nervous system, $\mathrm{H}^{+}$, ATP, $\mathrm{Mg}^{2+}$ and reactive oxygen species. Fatigue has been caused by such factors within the muscle cells and diminished activation from the central nervous system [1]. However, we have never been able to state why we experience skeletal muscle fatigue. The discussion following the work of Angelo Mosso stated that skeletal muscle fatigue involved the production of toxic substances such as carbonic acid on his collective studies [2].

Many researchers have reported that acupuncture therapy has been useful to muscle fatigue on various diseases and conditions. On multiple sclerosis, acupuncture showed the great improvement of Multiple Sclerosis Impact Scale 29 pyschological subscale [3]. However, it has never been shown why acupuncture therapy has the effect on muscle fatigue.

The purpose of this review is to demonstrate the effect of acupuncture on skeletal muscle fatigue.

\section{Acupuncture on Skeletal Muscle Fatigue}

Acupuncture has therapeutic effects on the skeletal muscle fatigue on various diseases and conditions. It has been showed that acupuncture reduces perceived pain arising from exercise-induced muscle soreness on randomized, controlled, observer and subjective-blinded trail. Pain perception 72 hours after acupuncture was significantly lower than control group [4]. Fibromyalgia has various symptoms as pain, debilitating fatigue, sleep disturbance, and joint stiffness. The one of them is skeletal muscle fatigue. The performance of acupuncture for 114 participants diagnosed with fibromyalgia has been carried out on a single-site, single-blind randomized trail. Acupuncture improvement in pain, fatigue and physical function were highly codependent in fibromyalgia [5].

We have often experienced skeletal muscle fatigue as low back pain. On systematic review of acupuncture for low-back pain, acupuncture has moderately effects, pain relief, and improvement of function from short-term. Acupuncture was moderately superior for long term pain relief [6-8]. Pain tolerance in the masticatory muscles increased significantly more with acupuncture. Acupuncture reduces significantly in face pain neck pain, and headache. Acupuncture peripheral stimulus can act as modular mechanism of muscle activity and was possible to verify correspondence of the auricular acupoint with the trapezius muscle [9]. Acupuncture was efficacious for improving isometric quadriceps strength in recreational athletes in a double-blind crossover trail. Acupuncture might have implications not only for athletes, but also for rehabilitation programs aimed at restoring neuromuscular function [10]. 
Acupuncture may become an additional therapeutic atrategy to improve the exercise tolerance of patients with congestive heart failure, potentially by improving skeletal muscle function. Acupuncture partially prevented skeletal muscle atrophy. This effect might be due to an increase in protein synthesis and a decrease in protein degradation [11].

These data showed that acupuncture have effects on the performance, strength, and fatigue of skeletal muscle.

\section{Carnitine of Skeletal Muscle}

On energy production, fatty acid has a main role. Mitochondria produce energy by uptake of fatty acid. But fatty acid arrives at the surface of mitochondoria, it is impossible to transport into mitochondoria membrane. Fatty acid consists on the activation to acyl CoA. This transport is accompanied by carnitine. Carnitine is a transporter of acetyl CoA into mitochondria.

An acute increase in human skeletal muscle total carnitine content results in an inhibition of carbohydrate oxidation in conditions of high carbohydrate availability. It is possibly due to a carnitine-madiated increase in fat oxidation. They have important implications for our understanding of the regulation of muscle fat oxidation, particularly during exercise. These results showed that carnitine availability may limit fat oxidation, and in obesity and type 2 diabetes [12,13].

Carnitine has an important role in energy production and modulation of the intramitochondrial coenzyme A (C0A)/acyl-CoA ratio in the skeletal muscle. The deficiency of carnitine might induce an energy deficit and abnormality of the intramitochondial condition in the skeletal muscle. These results show in general fatigue, myalgia, muscle weakness, and postexertional malaise in patients with chronic fatigue syndrome [14].

Intracellular mechanism regulating fat oxidation has been investigated in human skeletal muscle during exercise. The availability of free carnitine may limit fat oxidation during exercise with high muscle glycogen [15].

L-carnitine supplementation improves muscular performance. That is based on the role of L-carnitine in regulating aerobic metabolism. L-carnitine delays muscle fatigue by the reducing oxidative damage. The delay of muscular fatigue was evident from mean frequency, 50\% peak force of contraction, peak force of contraction. These results demonstrated that L-carnitine delays muscle fatigue by the reducing oxidative damage of intermittent hypoxia [16].

Carnitine has been used to enhance human exercise performance., Brass et al studied that the fatigue of isolated rat skeletal muscle strips was studoed in vitro, on the hypothesis that carnitine directly modify skeletal muscule function. As the incubation with propionyl-L-carnitine maximal contractile force, carnitine directly imp- roves the fatigue characteristics of muscle enriched in type I fibers directly [17].

Carnitine deficiency may be an important factor of dialysis-associated muscular symptom. Acetylcarnitine and propionylcarnitine showed beneficial effects on muscular fatigue of dialysis. Acetylcarnitine had main effect on mental fatigue and propionylcarnitine on general fatigue [18].

L-carnitine has an important role to cellular energy metabolism. Prolonged low-dose L-carnitine treatment can improve dialysis-associated muscle symptoms by restoring carnitine tissue levels and washing out acyl moieties [19]. Levocarnitine induced a reduction of total fat mass, increases total muscular mass, and facilitates an increased capacity for physical and cognitive activity by reducing fatigue and improving cognitive function [20].

These results demonstrated that the deficiency of carnitine induces fatigue, weakness, and disorder of skeletal muscle.

\section{Acupuncture on Carnitine}

Skeletal muscle disease are associated with contractures, craps muscle stiffness and deep muscle aching. These phenomena relate to skeletal muscle fatigue [21].

The pathogenesis of fatigue in skeletal muscle condition has the relation carnitine. Carnitine appears to improve force of skeletal muscle while stimulated in situ. This effect of carnitine is acute and stereospecific [22]. In energy metabolism, carnitine has a major role in the translocation of long-chain fatty acids into the mitochondrial matrix for subsequent $\beta$-oxidation, and in the regulation of the mitochondrial in skeletal muscle [23]. Acupunctures can protect cells from injury of acute spots and maintain the functions of mitochondria so as to delay fatigue, prolong working time of skeletal muscles [24].

Toda has reported the effect of acupuncture on carnitine in skeletal muscle [25]. The mice of acupuncture group by inserting $3 \mathrm{~mm}$ deep at right Zsusanli (ST36) and Jiexi (ST41) with sterilized disposable stainless steel acupuncture needles were fixed in the animal cage. The right Zsusanli (ST36) and Jiexi (ST41) were acupunctured, allowed for 15 minutes. The mice of normal control group were not both acupunctured and stimulated. Carnitine level in muscle tissue of acupuncture group was significantly higher than normal control group (Table 1).

Table 1. Effect of acupuncture on carnitine in skeletal muscle (mean \pm standard error, $\mu \mathrm{mol} / \mathrm{g}$ ).

\begin{tabular}{cc}
\hline \multicolumn{2}{c}{ Carnitine } \\
\hline Acupuncture group & $654.17 \pm 50.52^{*}$ \\
Normal control group & $346.67 \pm 53.41^{*}$ \\
${ }^{*} p<0.001$. &
\end{tabular}




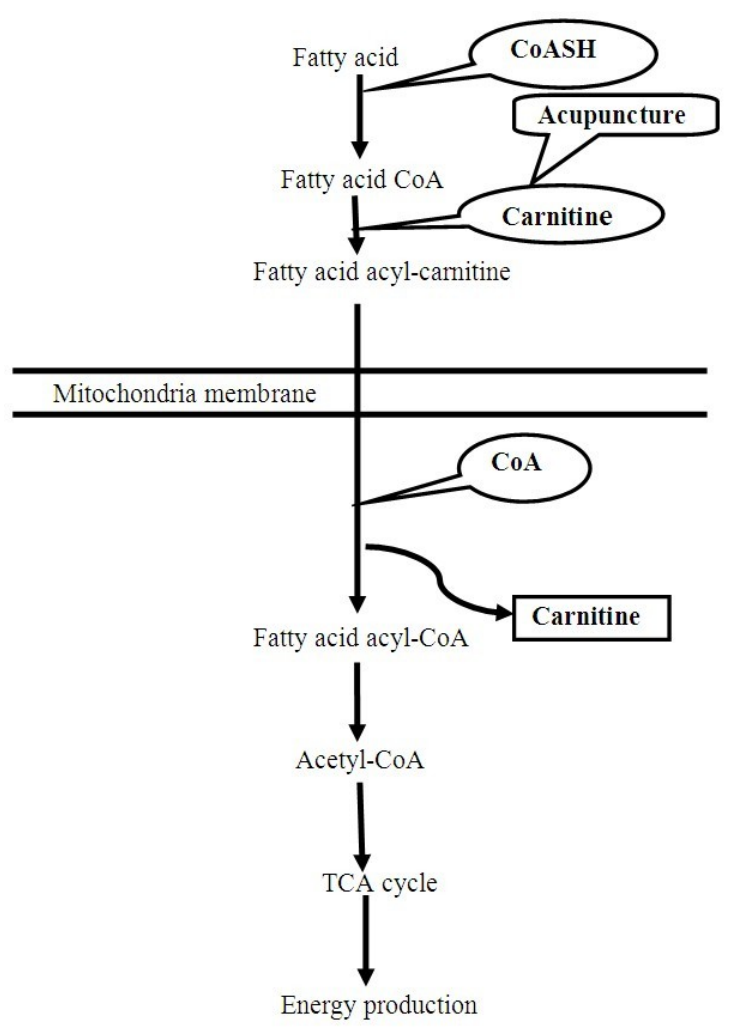

Figure 1. A model of acupuncture on carnitine and energy production.

Acupuncture at ST36 and ST41 has been shown to be a better therapeutic effect for muscle fatigue, problems of constriction and myofacial pain of leg muscle [26]. Acupuncture at these acupoints reduces the tibialis anterior electromyographymuscle activity [27].

Acupuncture leads to analgesia and improvement in other somatic sympotoms of fibromyalgia on a single-site, single-blind, randomized trial [28]. Acupuncture has been useful therapy on muscle symptoms. Carnitine therapy is effective in ameliorating fatigue in celiac disease on randomized double-blind versus placebo parallel study [29]. Carnitine can directly improve the fatigue characteristics of muscles enriched in type I fiber [30]. More than 95\% of carnitine in the body exists within skeletal muscle tissue [31]. Carnitine transfer fatty acids to the mitochondria, and involves energy metabolism. Carnitine deficiencies induce muscle fatigue and myopathy [32]. Carnitine in blood and brain showed to be increased by acupuncture [33].

These following findings showed that the effects of acupuncture may be related with carnitine. Acupuncture may be able to improve skeletal muscle fatigue by increasing carnitine in skeletal muscle (Figure 1).

\section{Conclusion}

Skeletal muscle fatigue is a common symptom experienced in various diseases, works and exercises. The defi- ciency of carnitine induces fatigue of skeletal muscle. It has showed that acupuncture induces the increase of carnitine in muscle. These findings demonstrated that acupuncture on skeletal muscle fatigue could increase carnitine as a possible affection mechanism.

\section{REFERENCES}

[1] D. G. Allen, G. D. Lamb and H. Westerblad, "Skeletal Muscle Fatigue: Cellular Mechanisms," Physiological Reviews, Vol. 88, No. 1, 2008, pp. 287-332. doi:10.1152/physrev.00015.2007

[2] D. Giulio, F. Daniele and C. M. Tipton, “Angelo Mosso and Muscular Fatigue: 116 Years after the First Congress of Physiologists: IUPS Commemoration," Advances in Physiological Education, Vol. 30, No. 2, 2006, pp. 51-57. doi:10.1152/advan.00041.2005

[3] C. P. Donnelan and J. Shanley, "Comparison of the Two Types of Acupuncture on Quality of Life in Secondary Progressive Multiple Sclerosis, a Preliminary SingleBlind Randomized Controlled Trial," Clinical Rehabilitation, Vol. 22, No. 3, 2008, pp. 195-205. doi:10.1177/0269215507082738

[4] M. Hubscher, L. Vogt, M. Bernhorster, A. Rosenhagen and W. Banzer, "Effects of Acupuncture on Symptoms and Muscle Function in Delayed-Onset Muscle Soreness," Journal of Alternative Complementary Medicine, Vol. 14, No. 8, 2008, pp. 1011-1016. doi:10.1089/acm.2008.0173

[5] R. F. Harris, X. Tian, D. A. Williams, T. X. Tian, T. R. Copps, F. Petzke, K. H. Groner, P. Biswas, R. H. Gracely and D. J. Clauw, "Treatment of Fibromyalgia with Formula Acupuncture: Investigation of Needle Placement, Needle Stimulation, and Treatment Frequency,” Journal of Alternative Complementary Medicine, Vol. 11, No. 4, 2005, pp. 663-671. doi:10.1089/acm.2005.11.663

[6] E. Manheimer, A. White, B. Berman, K. Forys and E. Ernst, "Meta-Analysis: Acupuncture for Low Back Pain," Annual Internal Medicine, Vol. 142, 2005, pp. 651-663.

[7] A. D. Furlan, M. van Tulder, D. Cherkin, H. Tsukayama, L. Lao, B. Koes, et al., "Acupuncture and Dry-Needling for Low Back Pain: An Updated Systematic Review within the Framework of the Cochrane Collaboration," Spine, Vol. 30, No. 8, 2005, pp. 944-963. doi:10.1097/01.brs.0000158941.21571.01

[8] A. D. Furlan, M. W. van Tulder, D. C. Cherkin, H. Tsukayama, L. Lao, B. W. Koes, et al., "Acupuncture and Dry-Needling for Low Back Pain,” Cochrane Database Systematic Review, Vol. 25, No. 1, 2005, CD001351.

[9] F. Politti, M. Vitti, C. F. Amorim, D. O. Tosello and E. T. Palomari, "Correspondence of the Auricular Acupoint with the Upper Trapezius Muscle: A Electromyographic Study," Complementary Therapeutic Clinical Practice, Vol. 16, No. 1, 2010, pp. 26-30. doi:10.1016/j.ctcp.2009.06.014

[10] M. Hubscher, L. Vogt, T. Ziebart and W. Banzer, "Immediate Effects of Acupuncture on Strength Performance: A Randomized, Controlled Crossover Trial," European 
Journal of Applied Physiology, Vol. 110, No. 2, 2010, pp. 353-358. doi:10.1007/s00421-010-1510-y

[11] A. Onda, Q. Jiao, Y. Nagano, T. Akimoto, T. Miyamoto, S. Minamisawa and T. Fukubayashi, "Acupuncture Smeliorated Skeletal Muscle Atrophy Induced by Hindlimb Suspension in Mice," Biochemical and Biophysical Research Communications, Vol. 410, 2010, pp. 434-439.

[12] F. B. Stephens, D. Constantin-Teodosiu, D. Laithwaite, E. J. Simpson and P. L. Greenhaff, "An Acute Increase in Skeletal Muscle Carnitine Content Alters Fuel Metabolism in Resting Human Skeletal Muscle," Journal of Clinical Endocrinology and Metabolism, Vol. 91, No. 12, 2006, pp. 5013-5018. doi:10.1210/jc.2006-1584

[13] F. B. Stephens, D. Constantin-Teodosiu and P. L. Greenhaff, "New Insights Concerning the Role of Carnitine in the Regulation of Fuel Metabolism in Skeletal Muscle,” Journal of Physiology, Vol. 581, 2007, pp. 431-444. doi:10.1113/jphysiol.2006.125799

[14] H. Kuratsune, K. Yamaguti, M. Takahashi, H. Misaki, S. Tagawa and T. Kitani, "Acylcarnitine Deficiency in Chronic Fatigue Syndrome," Clinical Infectious Diseases, Vol. 18, No. 1, 1994, pp. S62-S67. doi:10.1093/clinids/18.Supplement_1.S62

[15] C. Roepstorff, N. Halberg, T. Hilling, A. K. Saha, N. B. Ruderman, J. F. P. Wojtaszewski, E. A. Richter and B. Kiens, "Malonyl-CoA and Carnitine in Regulation of Fat Oxidation in Human Skeletal Muscle during Exercise," American Journal of Physiological Endocrinology and Metabolism, Vol. 288, No. 1, 2004, pp. E133-E142. doi:10.1152/ajpendo.00379.2004

[16] A. Dutta, K. Ray, V. K. Singh, P. Vats, S. N. Singh and S. B. Singh, "L-Carnitine Supplementation Sttenuates Intermittent Hypoxia-Induced Oxidative Stress and Delays Muscle Fatigue Inrats,” Experimental Physiology, Vol. 93, 2008, pp. 1139-1146. doi:10.1113/expphysiol.2008.042465

[17] E. P. Brass, A. M. Scarrow, L. J. Ruff, K. A. Masterson and E. Van Lunteren, "Carnitine Delays Rat Skeletal Muscle Fatigue in Vitro," Journal of Applied Physiology, Vol. 75, 1993, pp. 1595-1600.

[18] W. Vermeulen, R. Hans and R. Scholte, "Exploratory Open Label, Randomized Study of Acetyl- and Propionylcarnitine in Chronic Fatigue Syndrome,” Psychosomatic Medicine, Vol. 66, No. 2, 2004, pp. 276-282. doi:10.1097/01.psy.0000116249.60477.e9

[19] Y. Sakurauchi, Y. Matsumoto, T. Shinzato, I, Takai, Y. Nakamura, M. Sato, S. Nakai, M. Miwa, H. Morita, T. Miwa, I. Amano and K. Maeda, "Effects of L-Carnitine Supplementation on Muscular Symptoms in Hemodialyzed Patients," American Journal of Kidney Disease, Vol. 32, No. 2, 1998, pp. 258-264. doi:10.1053/ajkd.1998.v32.pm9708610

[20] M. Malaguarnera, L. Cammalleri, M. P. Gargante, M. Vacante, V. Colonna and M. Motta, "Carnitine Treatment
Reduces Severity of Physical and Mental Fatigue and Increases Cognitive Functions in Centenarians: A Randomized and Controlled Clinical Trial," American Journal of Clinical Nutrition, Vol. 86, 2007, pp. 1738-1744.

[21] H. Anthony, V. Schapira and C. Robert, "Muscle Pain and Fatigue. Muscle in Diseases,” Butterworth Heinemann, Boston, 1999, pp. 33-37.

[22] M. L. Dubelaar, C. M. Lucas and W. C. Hulsmann, "Acute Effect of L-Carnitine on Skeletal Muscle Force Tests in Dog," American Journal of Physiology, Vol. 260, 1991, pp. E189-E193.

[23] D. G. Allen, G. D. Lamb and H. Westerblad, "Skeletal Muscle Fatigue: Cellular Mechanism,” Physiological Review, Vol. 88, No. 1, 2008, pp. 287-332. doi:10.1152/physrev.00015.2007

[24] M. Gao, H. Y. Yang, T. Y. Liu and I. Kuai, "Effects of Manual Acupuncture and Electroacupuncture on Mitochondria of Skeletal Muscle Cells in Rats of Acute Swimming Exercise," Chinese Acupuncture \& Moxibustion, Vol. 25, 2005, pp. 421-424.

[25] S. Toda, "Investigation of Electroacupuncture and Manual Acupuncture on Carnitine and Glutathione in Muscle," Evidence-Based Complementary and Alternative Medicine, 2009, pp. 1-3.

[26] H. Gellman, “Acupuncture Physical Medicine,” Taylor \& Francis, Boston, 2002, pp. 162-180.

[27] L. A. Costa and J. E. Araujo, “The Immediate Effects of Local and Adjacent Acupuncture n the Tibialis Anterior Muscle: A Human Study," Chinese Medicine, Vol. 3, 2008, pp. 1-6. doi:10.1186/1749-8546-3-17

[28] R. E. Harris, X. Tian, D. A. Williams, et al., "Treatment of Fibromyalgia with Formula Acupuncture: Investigation of Needle Placement, Needle Stimulation, and Treatment Frequency," Journal of Alternative Complementary Medicine, Vol. 11, No. 4, 2005, pp. 663-671. doi:10.1089/acm.2005.11.663

[29] C. Ciacci, G. Peluso, E. Iannoni, et al., "L-Carnitine in the Treatment of Fatigue in Adult Celiac Disease Patients: A Pilot Study," Digestive and Liver Disease, Vol. 39, No. 10, 2007, pp. 922-928. doi:10.1016/j.dld.2007.06.013

[30] E. P. Brass, A. M. Scarrow, L. J. Ruff, et al., "Carnitine Delays Rat Skeletal Muscle Fatigue in Vitro,” Journal of Applied Physiology, Vol. 75, 1993, pp. 1595-1600.

[31] E. P. Brass, "Pharmacokinetic Consideration for the Therapeutic Use of Carnitine in Haemodialysis Patients," Clinical Therapy, Vol. 17, No. 2, 1995, pp. 176-185. doi:10.1016/0149-2918(95)80017-4

[32] C. Jacob and F. Belleville, "L-carnitine: Metabolism, Functions and Value in Pathology," Pathological Biology, Vol. 40, 1992, pp. 910-919.

[33] S. Toda, "Effects of Electroacupuncture on Carnitine in Serum and Brain," World Journal of Acupuncture-Moxibustion, Vol. 17, 2007, pp. 22-24. 